\title{
EXACT LOWER AND UPPER BOUNDS ON THE INCOMPLETE GAMMA FUNCTION
}

\section{IOSIF PINELIS}

Abstract. Lower and upper bounds $B_{a}(x)$ on the incomplete gamma function $\Gamma(a, x)$ are given for all real $a$ and all real $x>0$. These bounds $B_{a}(x)$ are exact in the sense that $B_{a}(x) \underset{x \downarrow 0}{\sim} \Gamma(a, x)$ and $B_{a}(x) \underset{x \rightarrow \infty}{\sim} \Gamma(a, x)$. Moreover, the relative errors of these bounds are rather small for other values of $x$, away from 0 and $\infty$.

Mathematics subject classification (2010): 33B20, 26D07, 26D15.

Keywords and phrases: Incomplete gamma function, exact bounds, inequalities, Gautschi inequalities.

\section{REFERENCES}

[1] Mathematical Reviews, Review MR0103289, 1960., https://mathscinet . ams . org/mathscinet-getitem? mr=MR0103289.

[2] H. AlZER, On some inequalities for the incomplete gamma function, Math. Comp., 66 (218): 771$778,1997$.

[3] H. AlZER AND A. BARICZ, Functional inequalities for the incomplete gamma function, J. Math. Anal. Appl., 385 (1): 167-178, 2012.

[4] G. E. Andrews, R. Askey, And R. Roy, Special functions, volume 71 of Encyclopedia of Mathematics and its Applications, Cambridge University Press, Cambridge, 1999.

[5] J. M. BORWEIN AND O.-Y. CHAN, Uniform bounds for the complementary incomplete gamma function, Math. Inequal. Appl., 12 (1): 115-121, 2009.

[6] W. GAuTSCHI, Some elementary inequalities relating to the gamma and incomplete gamma function, J. Math. and Phys., 38: 77-81, 1959/60.

[7] W. Gautschi, Personal communication, 2019.

[8] P. GREENGARD AND V. ROKHLIN, An algorithm for the evaluation of the incomplete gamma function, Adv. Comput. Math., 45 (1): 23-49, 2019.

[9] A. LAFORGiA AND P. NATAlini, Supplements to known monotonicity results and inequalities for the gamma and incomplete gamma functions, J. Inequal. Appl., pages Art. ID 48727, 8, 2006.

[10] P. Natalini And B. Palumbo, Inequalities for the incomplete gamma function, Math. Inequal. Appl., 3 (1): 69-77, 2000.

[11] E. NEUMAN, Inequalities and bounds for the incomplete gamma function, Results Math., 63 (3-4): 1209-1214, 2013.

[12] R. B. PARIS, Error bounds for the uniform asymptotic expansion of the incomplete gamma function, J. Comput. Appl. Math., 147 (1): 215-231, 2002.

[13] I. PinElis, L'Hospital type rules for oscillation, with applications, JIPAM. J. Inequal. Pure Appl. Math., 2 (3): Article 33, 24 pp. (electronic), 2001.

[14] I. PINELIS, On l'Hospital-type rules for monotonicity, JIPAM - J. Inequal. Pure Appl. Math., 7 (2): Article 40, 19 pp. (electronic), wWw. emis.de/journals/JIPAM/images/157_05_JIPAM/157_05.pdf, 2006.

[15] F. QI, Monotonicity results and inequalities for the gamma and incomplete gamma functions, Math. Inequal. Appl., 5 (1): 61-67, 2002.

[16] F. QI AND S.-L. GUO, Inequalities for the incomplete gamma and related functions, Math. Inequal. Appl., 2 (1): 47-53, 1999. 
[17] N. M. TEMmE, The asymptotic expansion of the incomplete gamma functions, SIAM J. Math. Anal., 10 (4): 757-766, 1979.

[18] Z.-H. YANG, W. ZHANG, AND Y.-M. CHU, Sharp Gautschi inequality for parameter $0<p<1$ with applications, Math. Inequal. Appl., 20 (4): 1107-1120, 2017. 